\title{
High field MR carotid vessel wall imaging: reproducibility of five different MR-weightings
}

\author{
Eleanore S Kroner ${ }^{1 *}$, Jos J Westenberg ${ }^{1}$, Rob J van der Geest ${ }^{1}$, Joost Doornbos ${ }^{1}$, Joanne D Schuiff ${ }^{1}$, Eline Kooi ${ }^{2}$, \\ Albert de Roos ${ }^{1}$, Jeroen J Bax ${ }^{1}$, Hildo J Lamb', Hans-Marc Siebelink ${ }^{1}$ \\ From 2011 SCMR/Euro CMR Joint Scientific Sessions \\ Nice, France. 3-6 February 2011
}

\section{Introduction}

Magnetic Resonance Imaging (MRI) has emerged as a promising noninvasive imaging modality for the serial assessment of vessel wall thickness in the carotid artery as an early marker of atherosclerosis. For clinical application of this technique, Scan-Rescan reproducibility is paramount. Currently, a multicontrast protocol, including a combination of MR-weightings is used as reference standard for quantitative and morphologic measurements.

\section{Purpose}

To investigate Scan-Rescan reproducibility for each of the commonly used weightings analyzed separately. To investigate which of the MR-weightings approximates best the combined multicontrast protocol (reference standard).

\section{Methods}

5 healthy volunteers $(60 \%$ male, mean age $=28$ years $)$ underwent repeated MRI examinations of the left carotid artery with five contrast-weighted scans to image lumen and vessel wall (Table 1). The scan and a rescan were acquired using a 3T (Philips) MRI scanner. A standard phased-array coil with two flexible elements of $14 \times 17 \mathrm{~cm}$ was used to obtain nine transverse imaging sections of the left carotid artery with identical in-plane resolution $\left(0.46 \times 0.46 \mathrm{~mm}^{2}\right)$. Scan-Rescan analysis was performed in the third slice of the imaging stack, representing a slice in the common carotid artery. An example is provided in Figure 1. Manual contour segmentation of the lumen and vessel wall was performed using in-house developed software (VesselMASS). Vessel wall area $\left(\mathrm{mm}^{2}\right)$ and lumen area $\left(\mathrm{mm}^{2}\right)$ were assessed

Table 1 Carotid Imaging Protocol at 3T: Scan Parameters

\begin{tabular}{|c|c|c|c|c|c|}
\hline Parameters & $\begin{array}{l}\text { Black-blood } \\
\text { T1-weighted }\end{array}$ & $\begin{array}{l}\text { Black-blood } \\
\text { T2-weighted }\end{array}$ & $\begin{array}{l}\text { Black-blood } \\
\text { Proton-Density-weighted }\end{array}$ & T1-weighted & TOF \\
\hline Acquisition sequence & TFE & TSE & TSE & TSE & FFE \\
\hline Acquisition Mode & $2 \mathrm{D}$ & $2 \mathrm{D}$ & $2 \mathrm{D}$ & $2 \mathrm{D}$ & $3 \mathrm{D}$ \\
\hline Echo Time (msec) & 3.54 & 50 & 20 & 10 & 3.30 \\
\hline Repetition Time (msec) & 12.41 & 2 heartbeats & 2 heartbeats & 1 heartbeat & 26.20 \\
\hline Excitation flip angle (degrees) & 45 & 90 & 90 & 90 & 20 \\
\hline FOV $(\mathrm{cm})$ & $14 \times 14$ & $14 \times 14$ & $14 \times 14$ & $14 \times 14$ & $14 \times 14$ \\
\hline Resolution (mm2) & $0.461 \times 0.461$ & $0.461 \times 0.461$ & $0.461 \times 0.461$ & $0.461 \times 0.461$ & $0.461 \times 0.461$ \\
\hline Slice thickness/gap (mm) & $2 ; 0.71$ & $2 ; 0.71$ & $2 ; 0.71$ & $2 ; 0.71$ & $2 ; 0.71$ \\
\hline Slices & 9 & 9 & 9 & 9 & 9 \\
\hline
\end{tabular}

TSE, turbo (segmented) spin-echo; FFE, fast field echo (gradient echo); TFE, turbo field echo; FOV, Field of view; TOF, time of flight

'LUMC, Leiden, Netherlands

Full list of author information is available at the end of the article 


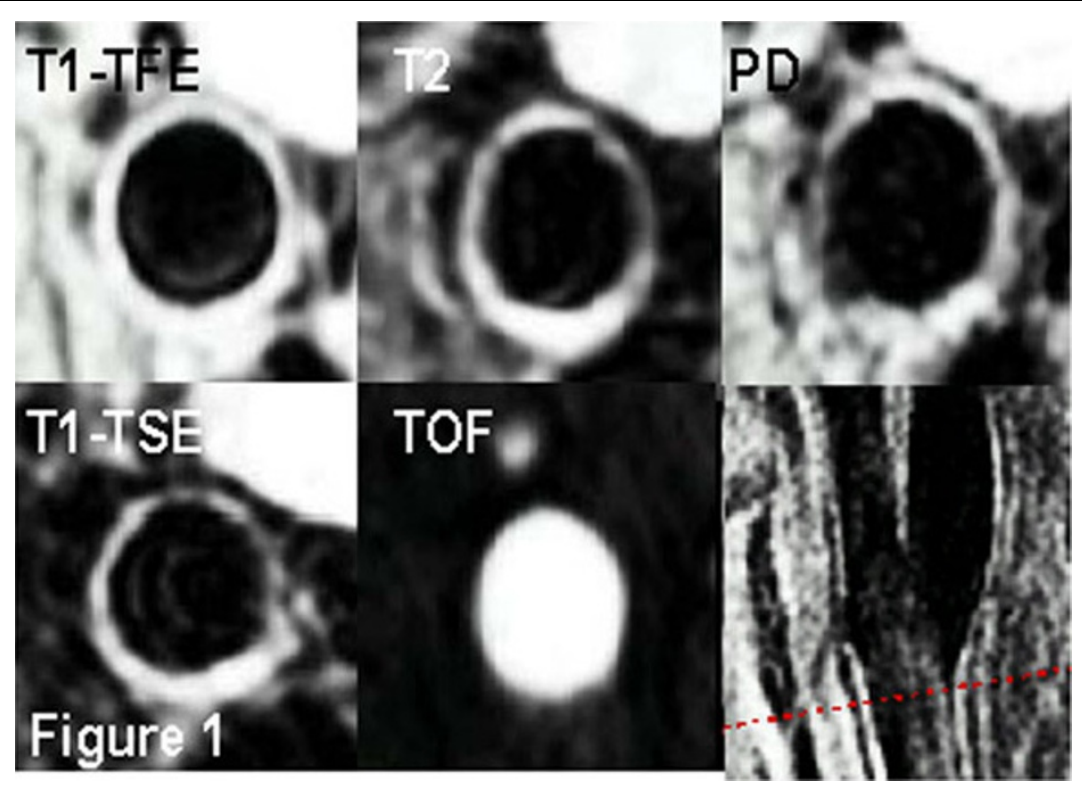

Figure 1 An example of the co-registered contrast-weightings and 3D time-of-flight. The red line on the sagittal view of the carotid bifurcation indicates position of the analyzed slices.

Table 2

\begin{tabular}{|c|c|c|c|c|c|c|c|c|c|c|}
\hline & \multicolumn{5}{|c|}{ Lumen Area (mm2) } & \multicolumn{5}{|c|}{ Vessel Wall area $(\mathrm{mm} 2)$} \\
\hline & $\mathbf{R}$ & mean & $p$ & SD & $\operatorname{cov}(\%)$ & $\mathbf{R}$ & mean & $p$ & SD & $\operatorname{COV}(\%)$ \\
\hline T1-TFE & 0.97 & -1.15 & 0.42 & 2.91 & $8 \%$ & 0.86 & 1.41 & 0.01 & 0.74 & $3 \%$ \\
\hline $\mathrm{T} 2$ & 0.95 & 2.03 & 0.18 & 2.82 & $8 \%$ & 0.96 & -1.19 & 0.20 & 1.72 & $7 \%$ \\
\hline PD & 0.89 & 2.75 & 0.06 & 2.28 & $7.5 \%$ & 0.55 & -0.25 & 0.85 & 2.82 & $12 \%$ \\
\hline T1-TSE & 0.90 & -0.12 & 0.94 & 3.45 & $10 \%$ & 0.85 & -1.70 & 0.13 & 2.03 & $9 \%$ \\
\hline TOF & 0.97 & 0.74 & 0.43 & 1.89 & $5 \%$ & & & & & \\
\hline \multicolumn{11}{|c|}{ b- Separate analysis of the MR-weightings compared to combined contrast weighted protocal } \\
\hline & \multicolumn{5}{|c|}{ Lumen Area (mm2) } & \multicolumn{5}{|c|}{ Vessel Wall area $(\mathrm{mm} 2)$} \\
\hline & $\mathbf{R}$ & mean & $\mathrm{p}$ & SD & $\operatorname{cov}(\%)$ & $\mathbf{R}$ & mean & $\mathrm{p}$ & SD & $\operatorname{COV}(\%)$ \\
\hline T1-TFE & 0.97 & 0.57 & 0.52 & 1.80 & $5 \%$ & 0.90 & -0.60 & 0.18 & 0.83 & $3.5 \%$ \\
\hline $\mathrm{T} 2$ & 0.38 & -0.76 & 0.85 & 8.32 & $24 \%$ & 0.52 & -1.02 & 0.42 & 2.54 & $11 \%$ \\
\hline PD & 0.81 & -2.71 & 0.24 & 4.39 & $13 \%$ & 0.07 & -0.50 & 0.75 & 3.31 & $14 \%$ \\
\hline T1-TSE & 0.78 & -0.69 & 0.75 & 4.63 & $13 \%$ & 0.64 & -2.25 & 0.15 & 2.87 & $13 \%$ \\
\hline TOF & 0.84 & 3.08 & 0.15 & 3.88 & $10 \%$ & & & & & \\
\hline
\end{tabular}

R. Pearson's correlation coefficient; P p-value of T-test; SD. Standard deviation; COV. Coefficient of variation.

by one blinded observer for the different contrast weightings and compared with the rescan acquisition. Furthermore, vessel wall- and lumen areas from the different contrast weightings were compared with the reference standard.

\section{Results}

Reproducibility of the repeated assessment was high for all MR-weightings, for both lumen area and vessel wall areas. (Table 2). The Bland-Altman plot for vessel wall area, is shown in Figure 2 for Scan-Rescan reproducibility. Highest reproducibility was found for the T1-TFE and T2-TSE sequences.

T1-TFE showed highest correlation for lumen $(r=0.97)$ and vessel wall area $(r=0.90)$ assessment when compared with the reference standard.

\section{Conclusion}

This pilot Scan-Rescan study showed best reproducibility of lumen and vessel wall area assessment for the 


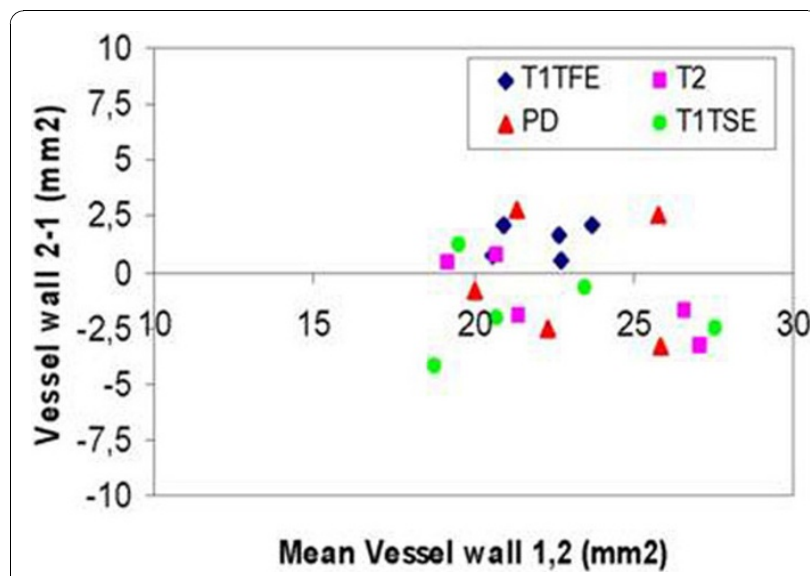

Figure 2 Bland-Altman Plot: Scan-Rescan Reproducibility (Vessel Wall Area).

T1-TFE and T2-TSE weightings. T1-TFE showed highest correlation to the reference standard.

Author details

'LUMC, Leiden, Netherlands. ${ }^{2}$ AZM, Maastricht, Netherlands.

Published: 2 February 2011

doi:10.1186/1532-429X-13-S1-013

Cite this article as: Kroner et al:: High field MR carotid vessel wall imaging: reproducibility of five different MR-weightings. Journal of Cardiovascular Magnetic Resonance 2011 13(Suppl 1):013.
Submit your next manuscript to BioMed Central and take full advantage of:

- Convenient online submission

- Thorough peer review

- No space constraints or color figure charges

- Immediate publication on acceptance

- Inclusion in PubMed, CAS, Scopus and Google Scholar

- Research which is freely available for redistribution

Submit your manuscript at www.biomedcentral.com/submit 